\title{
DER UMBAU DER UNIVERSITÄTSBIBLIOTHEK GRAZ - EIN BERICHT IN DREI FORTSETZUNGEN - TEIL 2: VOM FALL UND AUFSTIEG EINES GEBÄUDES
}

von Werner Schlacher

Zusammenfassung: Dieser Teil des Berichts vom Umbau der Universitätsbibliothek Graz beinhaltet die Phase vom Abriss des alten Gebäudes bis zur Gleichenfeier im Juli 2018.

Schlüsselwörter: Universitätsbibliothek Graz; Umbau

\section{THE RECONSTRUCTION OF THE UNIVERSITY LIBRARY OF GRAZ - A REPORT IN THREE SEQUELS. PART 2: ON THE FALL AND RISE OF A BUILDING}

Abstract: This part of the report contains the period from the demolition of the old building until the topping-out ceremony in July 2018.

Keywords: University Library of Graz; reconstruction

DOI: https://doi.org/10.31263/voebm.v71i3-4.2162

(C) Werner Schlacher

Dieses Werk ist lizenziert unter einer

Creative-Commons-Lizenz Namensnennung 4.0 International 


\section{„Das Alte stürzt...“}

Im Herbst 2016, als ich Teil 1 dieses Berichts ${ }^{1}$ verfasste, waren die einzelnen Abteilungen der Bibliothek in die vorgesehenen und entsprechend adaptierten Ersatzquartiere übersiedelt und hatten dort ihre Arbeit aufgenommen. Ein Zeitplan für die Bauarbeiten war bekannt, und obwohl die Behördenverfahren damals noch nicht abgeschlossen waren, konnte man berechtigterweise davon ausgehen, dass der vorhergesagte Abschluss des Vorhabens termingerecht im Frühsommer 2019 erfolgen würde. Schon kurz nach dem Abtransport der Buchbestände aus den einvernehmlich festgelegten Bereichen des Gebäudekomplexes und dem Auszug der Kollegenschaft samt der in den Ersatzquartieren weiterhin benötigten Büroeinrichtung wurde denn auch mit der Demontage von großen Anlagen und Installationen im Inneren begonnen. In wenigen Monaten wurden zahlreiche Regalanlagen, die Aggregate der Klimaanlagen, die Lifte etc. abgebaut und entsorgt.

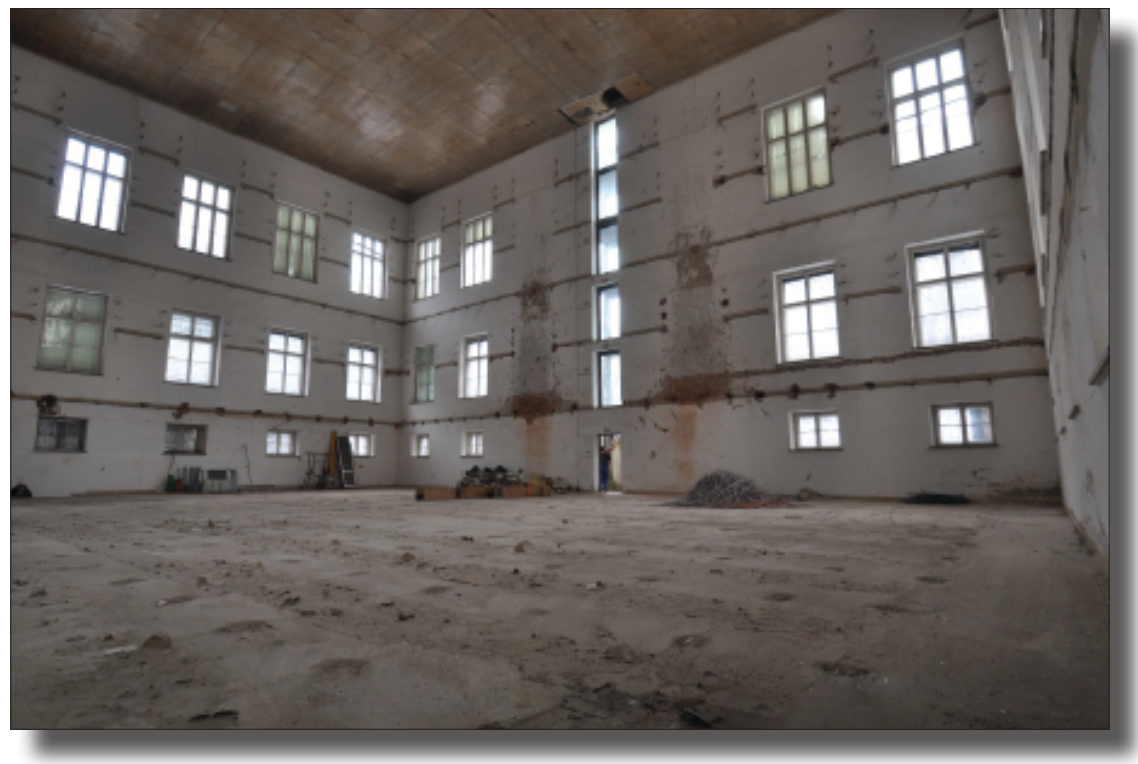

Abb. 1: Das alte Hauptmagazin ohne die Wertheim-Regalanlage (Foto: Robert Ramschak)

Die Katalogschränke, die nicht entsorgt werden konnten, weil die Finanzierung der mehrfach geplanten Retrokatalogisierung nie genehmigt worden war, mussten mangels anderer Möglichkeiten in den denkmalgeschützten Lesesaal verbracht werden. Gemeinsam mit dem beweglichen Mobiliar des 
Lesesaales wurde sie in einer Ecke zusammengestellt und abgedeckt, um eine allzu starke Verschmutzung zu verhindern. Der historische Raum wurde für die Zeit der Bautätigkeit generell geschlossen, er soll aber vor der Neueröffnung der Bibliothek generalsaniert und das gesamte Interieur einer vollständigen Restaurierung unterzogen werden. Um das Austrocknen des Holzes zu verhindern, wurde eine Beheizungs- und Befeuchtungsanlage installiert. Die Recherche in den Katalogsequenzen 1501-1925 und 19261982 ist seither nur über den online verfügbaren Imagekatalog möglich.

Im April 2017 begannen die spektakulären Abrissarbeiten an dem Anfang der siebziger Jahre errichteten Gebäudeteil im Norden des Lesesaales. Schon die Anlieferung des 70t schweren Raupenfahrzeuges, das in mehreren Teilen auf Sattelschleppern transportiert wurde und das sich in kurzer Zeit selbst zusammenbaute, war überaus eindrucksvoll. Schließlich begann der imposante Greifarm seine zerstörerische Tätigkeit und brachte Stück für Stück des unter Franz Kroller 1970 fertiggestellten Verwaltungstraktes der Bibliothek zum Einsturz.

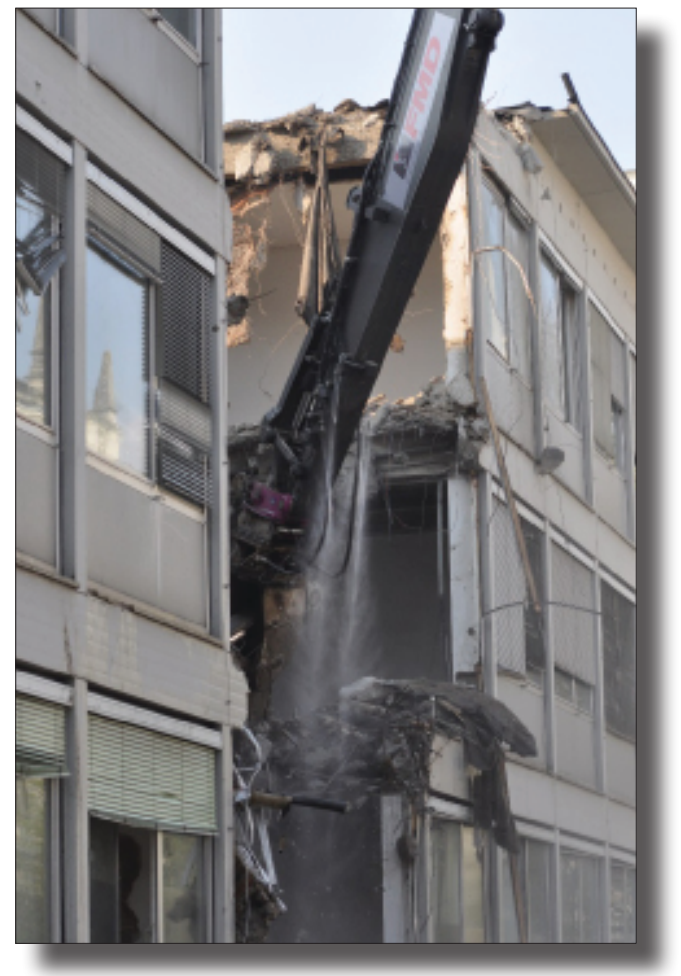

Abb. 2: Die ersten Mauern fallen (Foto: Robert Ramschak) 
Viele der KollegInnen, die das Voranschreiten der Arbeiten immer wieder mit großem Interesse beobachteten, beschlich ein wehmütiges Gefühl beim Anblick, wie das Büro, in dem sie oft jahrzehntelang ihren Tätigkeiten nachgegangen waren, in wenigen Minuten für immer verschwand. Im Laufe mehrerer Wochen drang der Greifarm immer weiter ins Innere des Gebäudes vor, bis endlich die aus den 1890er Jahren stammende, ursprüngliche Fassade des Lesesaaltrakts zum Vorschein kam, die nahezu fünfzig Jahre völlig verbaut und daher unsichtbar gewesen war.

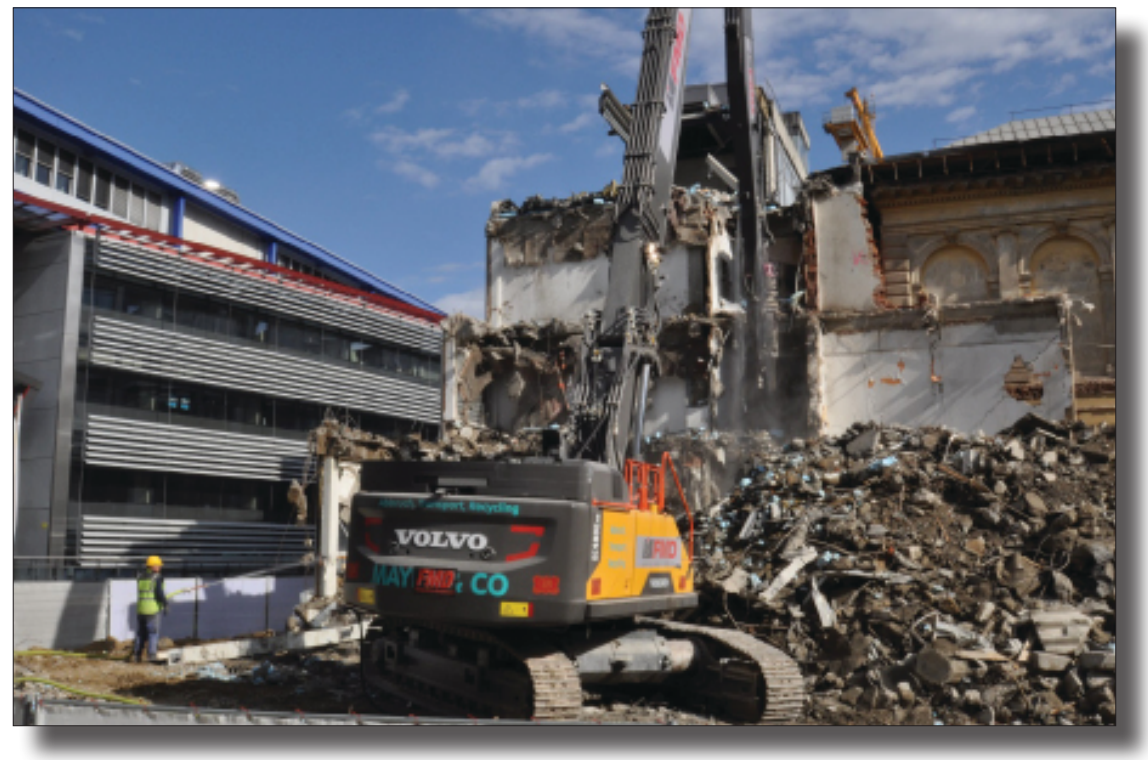

Abb. 3: Große Teile des alten Gebäudes sind nur mehr Schutt. Links das ReSoWi-Gebäude, rechts im Hintergrund die alte Fassade aus dem 19. Jahrhundert (Foto: Robert Ramschak)

Zu einem sehr bedauerlichen Zwischenfall kam es aus nach wie vor nicht geklärter Ursache im Mai 2017. Im Zuge der Bauarbeiten wurde eine Fußbodenheizungsanlage im Domenig-Eisenköck-Trakt ${ }^{2}$, deren Existenz niemanden bekannt war, beschädigt. Das auslaufende Wasser sickerte durch die Decke in den darunter befindlichen Vorraum eines in Betrieb befindlichen Magazins der Bibliothek, das - wie man uns versichert hatte - vom Baugeschehen gänzlich verschont bleiben sollte. Der Schaden wurde bald entdeckt und weil sich in diesem Vorraum keine Bücher befunden hatten, hielten sich die Auswirkungen in Grenzen. Da diese Fußbodenheizung im Ablauf des weiteren Baugeschehens ohnehin entfernt werden sollte, veranlasste die Bauleitung nur, dass das Zulaufventil geschlossen wurde. An 
einem Wochenende rund zwei Wochen danach jedoch wurde dieses Ventil von bis heute unbekannter Hand und zu ebenso unbekanntem Zweck wieder geöffnet, und das Wasser drang durch das Leck in der Heizungsanlage und die Kellerdecke diesmal in einen Raum ein, in dem wir in Absprache mit der Bauleitung die Nachschlagewerke aus dem Lesesaal vorübergehend aufbewahrten.

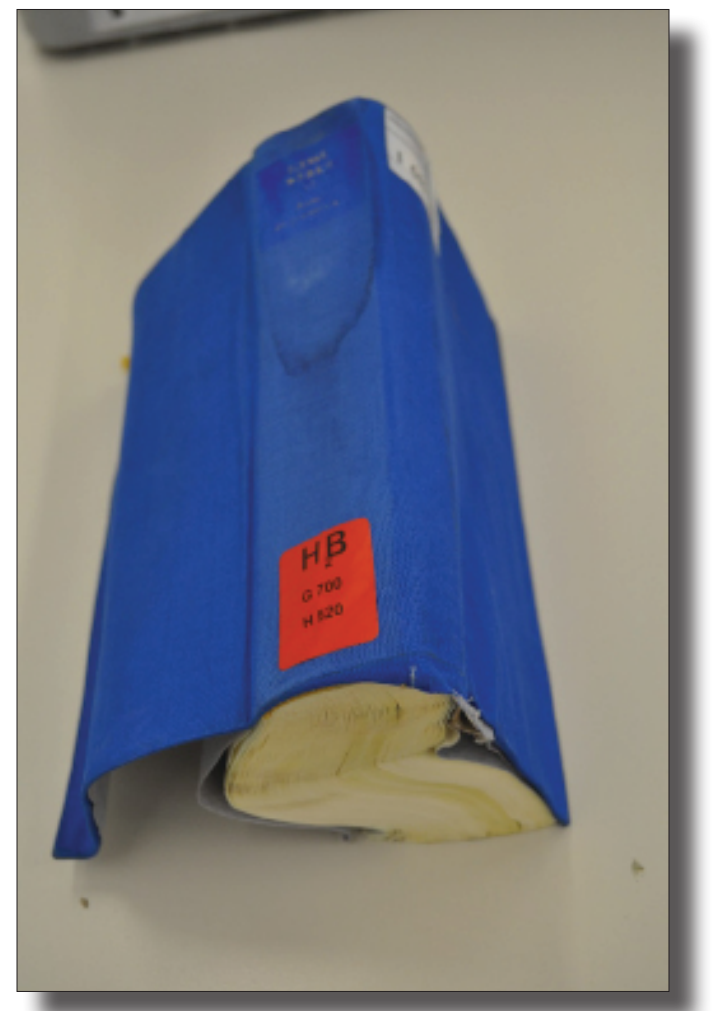

Abb. 4: Durch Wasserschaden völlig deformierter Band (Foto: Ramschak)

Wir wissen bis heute nicht genau, wie lange das Wasser auf die Regale rann, aber als der erste Bibliotheksmitarbeiter am Montag in der Früh ins Magazin kam, bot sich ihm ein erschreckendes Bild. In dem Raum, der mit einer Kompaktregalanlage ausgestattet war, floss das Wasser an mehreren Stellen ungehindert von der Decke auf die darunter befindlichen Werke, die zu diesem Zeitpunkt bereits so vollgesogen waren, dass sie die Stahlregale teilweise erheblich verbogen hatten. In Zusammenarbeit mit der sofort verständigten Firma Belfor wurde unverzüglich mit einer Notbergung der 
insgesamt 80.000 Bücher begonnen, wobei an den am ärgsten betroffenen Stellen, zuerst die Regale zerlegt werden mussten, bevor man die durch die Wasseraufnahme enorm schweren Bände entnehmen konnte. Es wurde rasch entschieden zu versuchen, die direkt vom Wassereintritt betroffenen Bestände (rund 10\% des betroffenen Gesamtbestandes) durch Gefriertrocknung vor dem endgültigen Untergang zu retten. Der Rest wurde von Mitarbeitern der Bibliothek in einen zwei Stockwerke darüber liegenden Raum verbracht und dort aufgefächert am Boden aufgestellt.

Leider konnte durch die sofort eingeleiteten Maßnahmen nicht verhindert werden, dass es in einigen Bänden aufgrund der Restfeuchtigkeit zu einer Schimmelbildung kam, die durch aufwendige Wischdesinfektion beseitigt werden musste. Der überwiegende Teil der gefriergetrockneten Bände wurde in weiterer Folge an Buchbinder zur weiteren Behandlung übergeben, bei einer kleineren Anzahl war eine Ersatzbeschaffung antiquarischer Exemplare kostengünstiger bzw. unumgänglich, da z.B. wegen zusammenklebender Seiten eine Wiederherstellung ohne gravierenden Informationsverlust nicht mehr möglich war.

Kurz nach dem Wassereintritt, aber in keinem kausalen Zusammenhang zu diesem, wurden die Bauarbeiten für mehrere Monate nahezu vollständig eingestellt. Der Grund dafür bestand in unterschiedlichen Ansichten über die statischen Anforderungen des Gebäudes. Soweit bekannt wurde, waren sich die Statiker nicht einig, in welchem Ausmaß das bestehende Mauerwerk aus dem 19. Jahrhundert in die Ableitung der durch die Aufstockung hinzukommenden Lasten einberechnet werden dürfe. Letztlich setzte sich die Meinung durch, dass das zusätzliche Gewicht der zwei neuen Stockwerke zur Gänze durch ein System von neu zu errichtenden Stahlsäulen und -trägern aufzufangen ist. Diese Entscheidung hatte weitgehende Neuplanungen und eine Verringerung des Platzangebots im Innenraum zur Folge. Die für Dezember 2017 fixierte Gleichenfeier musste wegen der Neuerstellung der Pläne auf Juni 2018 verschoben werden.

Trotz zum Teil noch nicht feststehender Grundrisse wurden im zweiten Halbjahr 2017 mit der Planung der Innenausstattung begonnen, wobei sich sehr rasch zeigte, dass die Bibliothek auch auf diesem Gebiet nur wenig Einfluss auf die Gestaltung der verschiedenen Gebäudebereiche nehmen können wird. So konnten Teppichböden in den Benutzerbereichen trotz mehrerer guter Gegenargumente ebenso wenig verhindert werden wie Kabelauslässe im Boden, die sich schon im alten Gebäude als unzweckmäßig erwiesen hatten und schließlich umgebaut werden mussten. Noch weitaus schwerwiegender jedoch ist die Tatsache zu bewerten, dass wegen einer plötzlich erforderlichen Vergrößerung der Querschnitte der Rohre für die Belüftung der oberen Stock- 
werke die für den Keller vorgesehene Regalanlage nur mehr mit fünf statt ursprünglich sechs Fachboden ausgestattet sein wird. Das bedeutet einen Verlust von rund siebzehn Prozent und bei einem geplanten Fassungsvermögen von einer Million Bände fehlenden Stellraum für 170.000 Bücher.

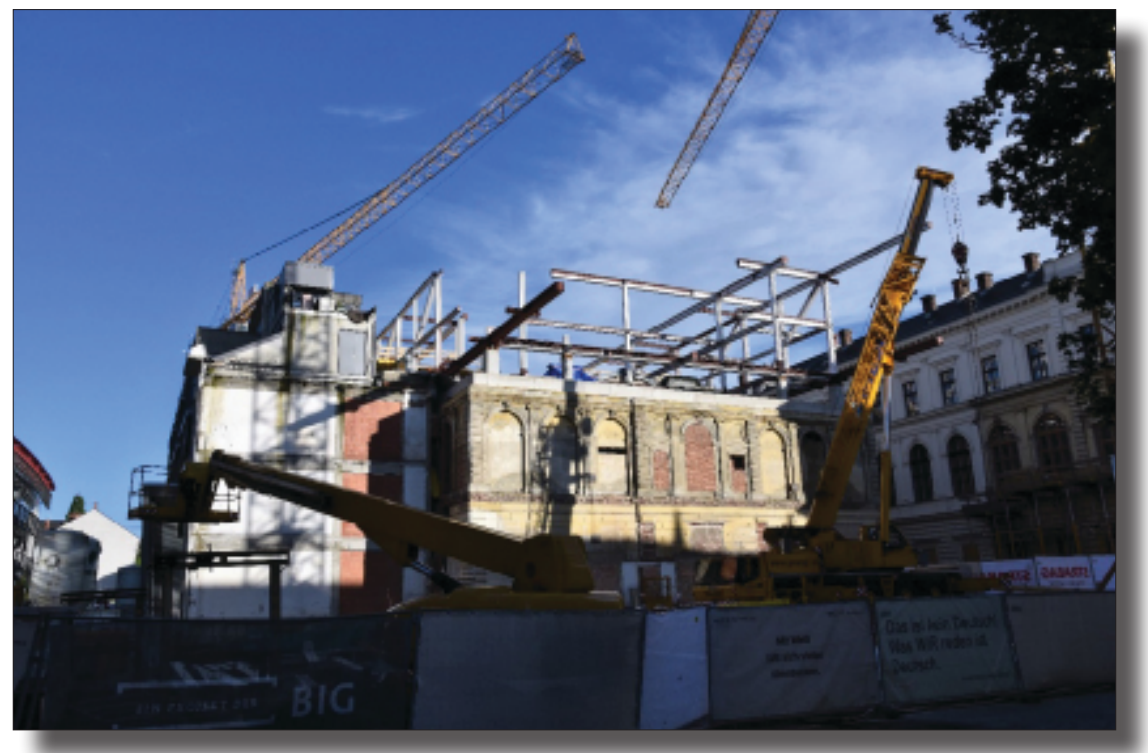

Abb. 5: Errichtung des Stahlskeletts für die Auskragung (Foto: R. Ramschak)

Im Jänner 2018 wurden die Bauarbeiten schließlich wiederaufgenommen und im darauffolgenden April mit der Errichtung des Stahlskeletts für das dritte und vierte Obergeschoß begonnen. Obwohl es wegen kolportierter Lieferschwierigkeiten der Stahlbaufirma zu einer neuerlichen Bauverzögerung kam und die Konstruktion bis zu diesem Termin bei weitem nicht abgeschlossen werden konnte, wurde die Gleichenfeier am 29. Juni 2018 durchgeführt.

Aus Sicht der Bibliothek stand im ersten Halbjahr 2018 vor allem die technische Ausstattung des neuen Gebäudes im Zentrum der Überlegungen. Neben den Regalanlagen für den Magazins- und den Freihandbereich, die ausgeschrieben und beauftragt wurden, mussten unter anderem die Planungen für die Buchförderanlage, die Buchsicherungsanlage, das Schließsystem und die Videoüberwachung weiterentwickelt werden. Definitive Entscheidungen betreffend die Ausführung und die Vergabe dieser für den Betrieb einer Bibliothek so wichtigen Ausstattungselemente konnten jedoch bislang nicht herbeigeführt werden. 


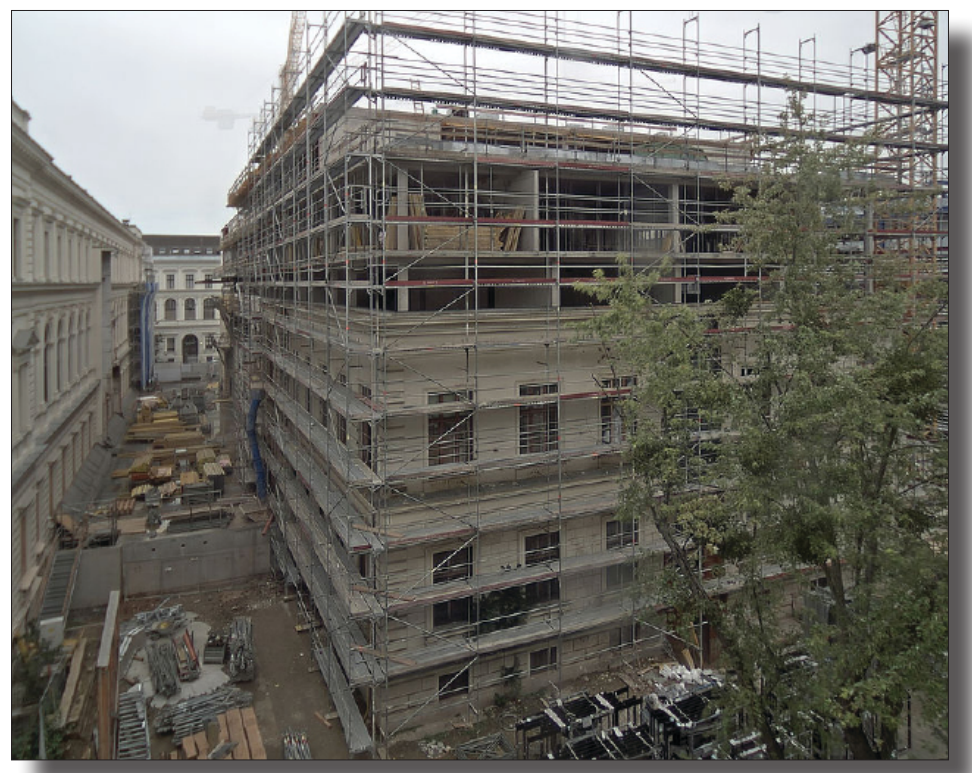

Abb. 6: Stand der Bauarbeiten von Süden gesehen am 4. September 2018; links das Universtiäts-Hauptgebäude, rechts die Bibliothek, dazwischen wird die neue Eingangshalle entstehen (Foto: Universität Graz)

Neben der Klärung unzähliger Detailfragen, die für die Fertigstellung und Inbetriebnahme des Baus unerlässlich ist und in die die VertreterInnen der Bibliothek mehr oder weniger eingebunden sind, wird in nahezu allen Diskussionen die Frage der Einhaltung des Zeitplans angesprochen. Nach aktueller Aussage einer offiziellen Vertreterin des Generalunternehmers wird der Fertigstellungstermin Sommer 2019 eingehalten. Der Österreichische Bibliothekartag von 10.-13. September 2019 in Graz bietet Ihnen eine gute Gelegenheit, sich selbst davon zu überzeugen.

Dr. Werner Schlacher Universität Graz, Universitätsbibliothek E-Mail: werner.schlacher@uni-graz.at

1 Teil 1 des Berichts erschien unter dem Titel „Der Umbau der Universitätsbibliothek Graz - ein Bericht in drei Fortsetzungen. Teil 1: Wie alles begann" in: Mitteilungen der Vereinigung Österreichischer Bibliothekarinnen und Bibliothekare 69 (2016) 3/4, S. 422-435. https://doi. org/10.31263/voebm.v69i3.1732 
2 Das ist jener Erweiterungsbau der Bibliothek, der gleichzeitig mit dem ReSoWi-Gebäude nach den Plänen von Günther Domenig und Hermann Eisenköck errichtet und 1996 eröffnet wurde. 